\title{
Pragmatics Encoded and Decoded Message
}

\author{
Valentina A. Gara, Edona H. Berisha \& Bashkim B. Gllareva \\ Correspondence: Miss. Valentina A. Gara, PR department, Prishtina International Airport JSC, Vrelle, Lipjan \\ 10070, Kosovo. E-mail: garavalentina@hotmail.com
}

Received: May 7, 2013 Accepted: June 14, 2013 Online Published: July 12, 2013

doi:10.5539/ells.v3n3p16 URL: http://dx.doi.org/10.5539/ells.v3n3p16

\begin{abstract}
People's utterances while interacting have different meanings according to the circumstances and the intentions that speakers try to convey to a listener, while the listener may misunderstand and even get insulted because of the lack of information regarding the subject. It is a field studied by Pragmatics but the main study of this paper is discussing the indispensible effort that should be made by speakers when conveying an idea by adding more details possible in order to prevent insulting. The paper deals with all features of Pragmatics including speech acts, reference and inference, presupposition and entailment, cooperation and implication, also discourse and culture. Various samples are given on each feature in the most appropriate way possible to present the cause of insults that may occur when people are interacting.
\end{abstract}

Keywords: encoded-speaker's belief, decoded-listener's belief, insult-impolite and offensive statement

\section{Introduction}

In all languages there are expressions that instead of conveying the intended meaning they bring up different perceptions and in most of the cases as a result of this they cause insults. Therefore this paper deals with two new terms to be used in Pragmatics in order to avoid the unintended insults. This paper brings two new terms to make the intended meaning easier understood. The new terms used for understanding the intended meanings are Pragmatics Encoded Message and Pragmatics Decoded Message in order to avoid insults. The terms Encoded and Decoded are not new in English language; they are used in general for referring to the information converted into symbols and the reversing process of how they are received by a receiver. However these two terms are used for different purposes in different fields including Information Technology, Mathematics, Army etc but Pragmatics Encoded Message and Pragmatics Decoded Message carry out different meanings other than the general use of them.

\section{Rationale}

This paper is focusing in using these two terms in field of Pragmatics with the attempts to explain that deixis and distance, reference and inference, presupposition and entailment and all what Pragmatics is concerned of can be grouped in two major terms:

\section{Pragmatics Encoded Message and}

Pragmatics Decoded Message

\section{Research Hypothesis}

According to the study of pragmatics literature the following hypotheses are proposed:

$$
\text { Hypothesis } 1
$$

The term Pragmatics Encoded Message describes the speaker's belief that his/her message is fully understood by a listener and the failure to reach this objective can result in insult

\section{Hypothesis 2}

The term Pragmatics Decoded Message describes the listener's belief that the information conveyed by a speaker shall be clearly understood and the failure to reach this objective can result in insult

\section{Research Question}

According to Yule 1996 'Pragmatics'-Pragmatics is concerned with the study of meaning as communicated by a speaker (or writer) and interpreted by a listener (or reader). Whereas according to http://en.wikipedia.org 
'Pragmatics is a subfield of linguistics which studies the ways in which context contributes to meaning'. There are numerous definitions of Pragmatics however we have taken only these two approaches to be discussed in this paper. Considering these two approaches and other approaches explaining Pragmatics the research question of this paper is: Is it possible to group all Pragmatic concerns within two major groups: the one of speakers' belief and of listener's belief of that intention?

\section{Pragmatics Encoded and Decoded Message - General Meaning}

The term Pragmatics Encoded Message is the speaker or writer's belief that what a speaker has in mind shall fully be correctly interpreted by a listener or reader. The term Pragmatics Decoded Message is the listener or reader's belief that the speaker's message shall convey the full information to be right interpreted by listener. There are two acronyms used here for Pragmatics Encoded and Decoded Message:

\section{Pragmatics Encoded Message - PEM}

Pragmatics Decoded Message - PDM

They can also be referred through symbols where $>+>$ is assigned for PEM denoting that the first sign $>$ is used for the first intention whereas the second sign $+>$ denotes the second possible intention. The symbol $>+<$ is assigned for PDM denoting that the first intention may be interpreted correctly whereas the second symbol+< denotes that it may be wrongly interpreted. PDM has another symbol denoting that the encoded message is interpreted as per speaker's intention and it comes as a result of $>+>/$. The last symbol (/) is used to denote that it is PDM and not PEM.

\subsection{Pragmatics Encoded and Decoded Message in Deixis}

The Deixis is a Greek term meaning 'pointing via language'. Therefore the deixis is further explained in terms of person's deixis, spatial deixis and temporal deixis. However PEM and PDM are used only in Person Deixis whereas the Spatial and Temporal deixis are fully conveying the message according to PEM and PDM.

PEM and PDM are used in proximal and distal forms.

\subsubsection{Person Deixis PEM and PDM}

Let's imagine that there are two girls dressed in blue standing close to the speaker and listener but they are totally different in height, weight etc. so the speakers intends to talk about the taller one so the PEM is taller one whereas the listener interprets it as he is talking about the short one.

Person deixis PEM PDM

She (the girl in blue) Stella Jennifer

Therefore from the above example we can see that in cases when two persons are having something in common which and only that is referred than the encoded message is wrongly interpreted because by referring to the wrong person it can result in insult. The PEM means the taller one which is his fiancé whereas the PDM refers to the short one and may direct any question inappropriately resulting insults.

$$
>+>\text { results }>+<
$$

whereas

$\begin{array}{lll}\text { Person deixis } & \text { PEM } & \text { PDM } \\ \mathrm{He} \text { (the old man) } & \text { Jack } & \text { Jack }\end{array}$

This example shows that the speaker's belief (PEM) is interpreted right according to the listener's belief (PDM).

$$
>+>\text { results }>+>\mid
$$

From the above examples except face-to-face interaction speaker and listener shall be aware of the same persons talking about and this need to be clarified through adjective phrases too:

Person deixis

She the taller one

\section{PEM PDM}

the taller one the taller one

So, instead of using only pronouns I, you, he, she, it - to fully convey and receive the meaning the PDM suggests that PEM shall use Adjective Phrases too.

Example regarding the social deixis which results insulting

Let's imagine that an environment and the Queen of England is present at a visit in a poor family so two children (one is aged 18-the age is used in the context of being able to help the mother) are sitting at the dining room and 
looking at the Queen. Therefore the mother prepares the coffee by herself and then she enters the room with cups of coffee and while placing the coffee on the table (in front of the Queen) she looks at her older daughter and makes the utterance:

\section{Social Deixis \\ PEM PDM}

Would her highness like some coffee?

Daughter Queen

Therefore it can result in a confusion and insult to the Queen. So PDM suggests that PEM shall use more direct way in such cases when such situations are present.

\subsection{Pragmatics Encoded and Decoded Message in Reference and Inference}

Considering reference the PEM and PDM can be also based on the invented names by people like: Mr. Aftershave (Yule 1996-Pragmatics). The PED and PDM in reference face inabilities to be fully accomplished due to the reasons that speaker's belief are not corresponding with listener's belief. In these cases if it is listener's assumption which reaches the successful reference what will happen when the assumption is totally wrong? If Mr. Aftershave is referred by a speaker as an invented name by a speaker and it is expected to be understood by a listener then in some situations it can be fully misunderstood and it won't reach its aims. For example: Let's imagine if a person brings milk every each day in a restaurant and the people working in that restaurant refer to him as Mr. Milk. Any of the employees could make the inference that he is referred as stupid or as Cow, and by depending on the listener's belief this referent name shall give additional information also for having a successful PED and PDM.

For example:

Reference

Man who delivers the milk to us is late today.

\section{PEM}

Man delivering the milk

$$
>+>\text { results }>+>/
$$

whereas

Reference

\section{PEM}

Man delivering the milk

Mr. Milk is here

\section{PDM}

Man delivering the milk

So the speaker's belief doesn't correspond with the listener's belief and it res

\section{PDM}

Stupid Man

$$
>+>\text { results }>+<
$$

Therefore we shall have PEM and PDM in place at the cases when no previous statement was made. Reference according to PEM and PDM is made when previous statements describe the entities and we refer to them later otherwise it can also cause insult.

\subsection{Pragmatics Encoded and Decoded Message in Presupposition and Entailment}

The action of presupposition is defined about the speaker's belief prior to making the utterance. Even though they can be deniable according to the PEM and PDM they shall also be surrounding with the additional expressions too. Let's imagine the situation while the speaker refers to a person as 'insane' who is an ex friend of the listener.

Presupposition

$$
\begin{array}{lc}
\text { PEM } & \text { PDM } \\
\text { Insane } & \text { Insane } \\
& >+>\text { results }>+>/
\end{array}
$$

$\mathrm{He}$ is insane.

This statement may be assumed by a listener that the person is really insane. This can result in insult regardless of what the previous utterances were. Therefore to lessen this potential insult even though it can be deniable it is better if PEM shows the personal belief other than making a statement which may be considered as insult by PDM. PEM and PDM suggest the use of words such as: I presuppose, I think, I assume that etc.

Presupposition

I assume that he is insane

$$
\begin{array}{ll}
\text { PEM } & \text { PDM } \\
\text { He is insane } & \text { He may or may not be insane } \\
>+>\text { results }>+< &
\end{array}
$$

So according to this even if the person referred is an ex friend of listener it will lessen insulting by considering that he is expressing a pre-supposition and not a direct accusation toward the person referred as insane. 


\subsection{Pragmatics Encoded and Decoded Message in Cooperation and Implicature}

If speakers and listeners respect four maxims of Quality, Quantity, Relevance and Manner then PEM and PDM are in accordance with each other. However the violation of these maxims also results in PEM and PDM insulting.

Examples:

Violation of Quality Maxim

The girl next door has stolen my jewelry

Violation of Quantity Maxim

She failed all exams at first year

Violation of Relevance Maxim

She did kill a bird when she was five

Violation of Manner Maxim

She speaks but she can't write well and

she is going to be the one on top

of the black list of the tennis players

\section{PEM}

I guess it was her

PEM

then she succeeded later

PEM

she is a judge now

PEM

she can't participate

in miss world
PDM

She is a thief

PDM

She is a bad student

PDM

she is a killer

PDM

She is not smart

Considering the above examples it results that in all cases the PDM can insult the person referred by PEM.

5.5 Pragmatics Encoded and Decoded Message in Speech Acts and Events, Politeness and Interaction, Conversation and Preference Structure

Since speech acts can function as directives, representatives, expressives, declarations and commissives the PEM and PDM are in full accordance with each other. Therefore the speaker and listener are aware of the message they are sending and receiving. Speech events avoid the insulting or the situation of being refused through pre-request, pre-announcement etc therefore PEM and PDM are also in accordance to each other. Politeness and Interaction is another linguistic interaction which avoids insulting through Face Saving Act, Mitigating Device, off records, Say nothing etc and therefore it has been seen that PEM and PDM are not in contradiction. Conversation and Interaction have also defined rules about which the speakers and listeners are aware of and due to this they are fully applying the PEM and PDM.

\subsection{Discourse and Culture}

Speaker and listener's belief of discourse in pragmatics are set to be based upon the background knowledge of one's culture. However considering that discourse in pragmatics is totally different from the discourse in other linguistic fields it is more than crucial that the cultural schema is applied. However using PEM and PDM will easy make the avoidance of insult even though without prior knowledge of one's background and culture.

For example:

Let's imagine a person went to India as a guest to an Indian Family and at the mean time he is looking to a girl in that house which brings the meal (rice) and puts it on the table. The guest is looking at her and doesn't start eating. So, the hostess makes the following utterance:

Cultural schemata

PEM

PDM

We don't use forks for rice

we eat using fingers

$$
\begin{aligned}
& \text { he is threatening me } \\
& \text { they use forks to kill } \\
& \text { I wasn't looking at her } \\
& \text { for any bad intention }
\end{aligned}
$$$$
>+>\text { results }>+<
$$

This could be insult to a listener and the PEM could avoid this if it uses different expressions:

Cultural schemata

We eat rice without forks

$$
\begin{array}{ll}
\text { PEM } & \text { PDM } \\
\text { we eat using fingers } & \text { I shall eat it using fingers } \\
\multicolumn{2}{c}{>+>\text { results }>+>/}
\end{array}
$$

The above examples are not because of the lack of knowledge of schemata but due to wrong Pragmatics 
Encoded Message or wrong speaker's belief.

\section{Conclusion}

The study of the new terms Pragmatics Encoded and Decoded Message present a lot of information needed to be applied due to the messages that can cause insulting. Therefore they are used to find the best way of avoiding this as much as possible. Generally, the study was intended to avoid the inappropriate meanings conveyed by speaker according to his/her own belief in interpretation by a listener's belief. The methodology process has been based on Pragmatics selected literature and failure and success of application of the new terms have been considered. However these two notions are not being applied in some Pragmatics concerns such as speech acts and events, politeness and interaction, conversation and preference structure since the speaker's beliefs are interpreted in accordance with the listener's beliefs. By using these two terms not all the Pragmatics concerns can be grouped in two major groups of Pragmatic Encoded Message and Pragmatics Decoded Message because not all of the concerns of Pragmatics are in violation of these terms. Although applications of these two terms are not necessary to be applied for understanding the Pragmatics but they are needful for the right interpretation of messages and additionally they may be a subject of critics and discussion by bringing up further studies in regards to the conveyed meanings of utterances. Further studies regarding these two terms can be attractive and prospectively useful for future application during the social interactions in our everyday life.

\section{References}

Barbara Cairns. (1991). Lund University Department of Linguistics. Working Papers, 38, 19-28.

Barbara H. Partee. (2009). Formal Semantics Lecture 8 MGU April 24, p. 1

George Yule. (1996). Pragmatics (2nd ed.). Oxford University Press.

Irene Heim. (1988). On the Projection Problem for Presupposition in Proceedings of the Second West Conference on Formal Linguistics. Stanford University Press.

Kristine H. Onishi, \& Gregory L. Murphy. (2002). LANGUAGE AND COGNITIVE PROCESSES, 17(2), 97-123. University of Illinois at Urbana-Champaign, IL, USA.

Mitchell S. Green. (2007). Reference, Empty Names and Implicature 441199 University of Virginia Charlottesville. CANADIAN JOURNAL OF PHILOSOPHY DP, 37(3), 419-448.

http://ifla.uni-stuttgart.de/institut/mitarbeiter/jilka/teaching/Pragmatics/p5_implicatures.pdf (January 20, 2013)

http://www.um.edu.mt/_data/assets/pdf_file/0016/125233/lin1280-lecture7-cooperation.pdf (January 20, 2013)

http://www.um.edu.mt/_data/assets/pdf_file/0013/123241/lin1280-lecture5-reference.pdf (January 24, 2013)

http://en.wikipedia.org/wiki/Cultural_schema_theory (January 25, 2013)

http://www.um.edu.mt/_data/assets/pdf_file/0018/122148/lin1280-lecture1.pdf (January 29, 2013)

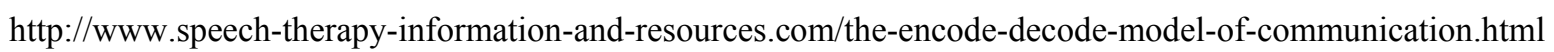

(February 01, 2013)

http://www.abc.net.au/science/holo/decode.htm (February 01, 2013)

http://www9.georgetown.edu/faculty/irvinem/theory/SH-Encoding-Decoding.pdf (February 04, 2013)

http://people.ucalgary.ca/ rseiler/hall.htm (February 04, 2013)

http://www.thefreedictionary.com/encode (February 04, 2013)

http://www.wikihow.com/Encode-and-Decode-Using-the-Vig\%C3\%A8nere-Cipher (February 04, 2013)

http://www.britannica.com (February 06, 2013)

http://www.um.edu.mt/_data/assets/pdf_file/0019/122149/lin1280-lecture2.pdf (February 26, 2013)

http://www.um.edu.mt/_data/assets/pdf_file/0011/122150/lin1280-lecture3.pdf (February 26, 2013)

http://www.coli.uni-saarland.de/courses/pragmatics-07/Slides/PD.07.4.ConversationStructure.pdf (February 26, 2013)

\section{Copyrights}

Copyright for this article is retained by the author(s), with first publication rights granted to the journal.

This is an open-access article distributed under the terms and conditions of the Creative Commons Attribution license (http://creativecommons.org/licenses/by/3.0/). 